Cárdenas-Rodríguez, R., Terrón-Caro, T. y Monreal Gimeno, M.C. (2019). Educación Primaria y alumnas gitanas. Análisis de las barreras sociales en contextos de exclusión. Revista de Investigación Educativa, 37(1), 75-91. DOI: http://dx.doi.org/10.6018/rie.37.1.326221

\title{
Educación Primaria y alumnas gitanas. Análisis de las barreras sociales en contextos de exclusión
}

\section{Primary education and romani girl students. Analysis of the social barriers in enviroments of exclusion}

\author{
Rocío Cárdenas-Rodríguez, Teresa Terrón-Caro y Mª Carmen Monreal Gimeno \\ Dpto. Educación y Psicología Social. Facultad Ciencias Sociales. Universidad Pablo de Olavide (España)'
}

\begin{abstract}
Resumen
Actualmente se está produciendo una transformación de los roles tradicionales asociados a hombres y mujeres, sin embargo, en los contextos desfavorecidos de exclusión social estas desigualdades entre hombres y mujeres sigue siendo muy notable. Este trabajo tiene como objetivo reflexionar sobre la situación de la mujer gitana en contextos desfavorecidos a lo largo de su proceso educativo, así como establecer las estrategias necesarias para favorecer el proceso educativo de estas alumnas. Para ello, aplicamos una metodología mixta basada en cuestionarios y grupos de discusión con profesorado y agentes claves de cuatro centros educativos situados en contextos de exclusión social con alto porcentaje de alumnado gitano. Los resultados obtenidos nos demuestran la percepción del profesorado y agentes claves sobre las dificultades de las alumnas gitanas en el Sistema Educativo, así como las actuaciones que se llevan a cabo desde los centros educativos para favorecer la progresión educativa de las alumnas gitanas. En cualquier caso, es importante señalar que el contexto socioeconómico determina la trayectoria educativa de las alumnas gitanas más que las características culturales, ya que muchas de las dificultades que se encuentran las alumnas están más relacionadas con los contextos de marginación social que con su cultura.

Palabras clave: cultura; educación; discriminación de género; diversidad étnica.
\end{abstract}

Correspondencia: Rocío Cárdenas-Rodríguez, mrcarrod@upo.es, Universidad Pablo de Olavide Departamento de Educación y Psicología Social. Edificio 11. Ctra. de Utrera, km. 1. 41013. Sevilla.

1 Estudio financiado por el Proyecto GENDERCIT (Género y Ciudadanía) coordinado por la Universidad Pablo de Olavide y financiado por el Programa People de la Unión Europea, IRSES, una de las acciones del Marie Curie, séptimo programa marco (FP7/2007-2013/under grant agreement 31896. 


\begin{abstract}
Nowadays a transformation in the traditional roles associated to men and women is being produced, however, in disadvantaged contexts of social exclusion these inequalities between men and women are still very notable. The following work aims to reflect on the situation of romani female in disadvantaged contexts throughout their educational process and to establish the necessary strategies to promote the educational process of these students. To do it, we applied a mixed methodology based on questionnaires and focus groups with teachers and key agents from four schools located in contexts of social exclusion with high percentage of romani students. The results obtained show us the perception of teachers and agents about the difficulties of romani female students in the Educational System, and the actions that are carried out from schools to promote the educational progression of romani female students. In any case, it is important to point out that the socio-economic context determines the educational trajectory of romani women students more than the cultural characteristics, since many of the difficulties encountered by the students are more related to the contexts of social marginalization than to their culture.
\end{abstract}

Keywords: Culture; Education; Gender Discrimination; Ethnic Diversity.

\title{
Introducción
}

La cultura gitana lleva presente en España desde antes del Siglo XV y ha estado marcada por persecuciones, intentos de asimilación y procesos de exclusión social. Aunque con la democracia y la lucha activa de las asociaciones en defensa del pueblo gitano se han dado grandes avances para la integración de este colectivo, tal y como indican Macías y Redondo (2012, p. 72), “(...) la realidad actual es que todavía no se ha conseguido superar la marginalización de la mayoría de este grupo", en muchas ocasiones invisibilizado por las diversas políticas desarrolladas tal y como indica Fernández Enguita (citado en El-Habib Draoui, Jiménez-Delgado, Ruiz-Callado, \& Jareño-Ruiz, 2015) con el fin de ocultar las desigualdades imperantes en relación a dicho colectivo.

En España, la escolarización del alumnado gitano en educación primaria se ha alcanzado casi al 100\%, y en educación infantil se han realizado grandes esfuerzos teniendo como resultado un aumento en el porcentaje de niños/as gitanos/as que asisten a la educación infantil siendo del 87\% en el año 2009 (Fundación para el Secretariado Gitano, 2010). Según un estudio realizado por la Fundación para el Secretariado Gitano (2013, p.8) “Hoy en día, la práctica totalidad de los niños y niñas gitanos acceden a la escuela a la edad obligatoria y los jóvenes gitanos están alcanzando niveles de instrucción superiores a los de sus padres y abuelos". En ese sentido podemos afirmar que la escolarización de los/as niños/as gitanas está prácticamente normalizada, esto se debe, en cierto modo, a la vinculación que han realizado las autoridades entre la escolarización y el acceso a determinados beneficios, ya sean bienes, servicios o transferencias de servicios público (Llevot \& Bernad, 2016). Por ello, aunque están escolarizados, nos encontramos con altos porcentajes de absentismo escolar y abandono prematuro, intensificándose estos porcentajes en el caso de las 
niñas gitanas y en educación secundaria (Ministerio de Sanidad, Servicios Sociales e Igualdad [MSSSI], 2012). Esto justifica la desigualdad que sigue existiendo, pues aunque exise un $100 \%$ de escolarización, no se ha producido "un incremento proporcional del nivel educativo y professional" (Parra, Alvarez-Roldan, \& Gamella, 2017, p. 38).

Por lo tanto, uno de los retos actuales que tiene la escuela, en relación con el alumnado gitano, es su permanencia y continuidad en el sistema educativo. Para ello, es fundamental que atienda a la distribución de los/las estudiantes gitanos/ as, favoreciendo su participación y cooperación en el aula tal y como indica Allport (citado en Sánchez-Muros, 2015). La escuela es un motor de cambio social ya que al conseguir la escolarización de los/as niños/as gitanas y una mayor permanencia en el sistema educativo se están produciendo cambios en los patrones familiares considerando cada vez más importante la educación de sus hijos/as, así como un cambio en las expectativas futuras de sus hijos/as. De hecho, según el MSSSI (2012), el absentismo escolar y el abandono prematuro no es acusado en la etapa de primaria, pero se acentúa en la etapa de secundaria donde el abandono sobre todo femenino sigue siendo muy alto, y en las etapas postobligatorias donde la presencia de alumnado gitano es muy escasa en comparación con la población general. Es fundamental disminuir dichas cifras ya que el abandono de la escuela por motivos culturales, prevee un futuro desigual con respecto al resto de la población tal y como indica Escudero (citado por Rodríguez \& Paterna, 2016).

En el año 2012, se publica en España el Plan Estratégico para la Inclusión Social de la Población Gitana en España 2012-2020², donde se presenta la educación como una de las líneas estratégicas de motor de cambio y transformación social, junto con el empleo, la sanidad, etc. Los objetivos de esta línea estratégica se centran en el acceso a la educación del alumnado gitano en educación infantil (no obligatorio); escolarización, permanencia y éxito del alumnado gitano en educación primaria; finalización del alumnado gitano de la etapa secundaria; e incrementar el nivel formativo de la población gitana adulta. A su vez, se considera prioritario no sólo el acceso al sistema educativo, sino también trabajar por la permanencia y el éxito del alumnado gitano para poder alcanzar la integración social. En esta línea, se debe indicar que, "a pesar de las medidas propuestas orientadas al desarrollo de estrategias de integración de aquellos colectivos más excluidos, no se ha otorgado la misma importancia en la superación de las desigualdades por razones de género" (Asensio, 2016, p.2), cuestión fundamental a tener en cuenta en cualquier colectivo y en especial en la población gitana.

En relación a las mujeres gitanas, además del sexismo sufren la discriminación social y cultural por pertenecer al colectivo gitano, por lo que tienen que luchar contra los prejuicios sexistas, culturales y sociales, y las barreras que han de superar en el

2 Esta estrategia se deriva de la Comunicación de la Comisión Europea de 5 de abril de 2011 al Parlamento Europeo, al Consejo, al Comité Económico y Social y al Comité de las Regiones "Un marco europeo de estrategias nacionales de inclusión de los gitanos hasta 2020" (COM (2011) 173 final). En dicha Comunicación se instaba a los Estados miembros a aprobar Estrategias nacionales para la inclusión de la población Roma/gitana (o un conjunto integrado de medidas). Las estrategias debían estar concebidas en coordinación con la Estrategia Europea 2020 y los Planes Nacionales de Reforma de cada país. 
ámbito formativo son mucho mayores, por lo que se encuentran en zona de vulnerabilidad y con un alto riesgo de caer en la exclusión social, por ello, autores como Márquez y Padua (citado en Márquez, Prados, \& Padua, 2017) plantean la necesidad de modificar los centros educativos para favorecer la inclusión y continuidad de las adolescentes gitanas, atendiendo a diversas dimensiones que permitan afrontar las barreras a las que deben hacer frente durante todo el proceso educativo.

Según Ayala (2014), desde la intervención social se considera a la mujer gitana como el verdadero "motor de cambio" de su comunidad, debido al proceso de empoderamiento que se ven obligadas a desarrollar ante la desigualdad a la que se encuentran sometidas (Zugaza, 2017). Son consideradas como referente de cambio participando activamente como mediadoras sociales ya que son las que presentan mayor deseo de mejora y mayor apertura al trabajo comunitario, son un referente en el cambio de la cultura gitana, son las más participativas y las que están transformando su realidad sin dejar de ser mujeres gitanas. Sin embargo, esta autora nos señala que "mientras a nivel público se consideran a las mujeres gitanas como agentes de cambio social, a nivel privado (en la esfera familiar) se las concibe como la parte débil y oprimida del colectivo gitano" (Ayala, 2014, p. 795). Por ese motivo, es importante que las mujeres gitanas estén presentes en cualquier espacio fuera del ámbito familiar como es el caso de la escuela, ya que favorece su socialización. Asimismo, debido al rol que adquiere en la familia como agente enculturador principal (López \& Sanz, 2017), es fundamental promover dicha transformación.

En el presente estudio analizamos cuales son las barreras y dificultades que se encuentran las alumnas gitanas en su proceso educativo, qué funcionalidad tiene la educación de sus hijas para sus familias, cuál es la relación de la familia con el centro educativo y qué estrategias educativas son necesarias desarrollar en los centros educativos para el progreso educativo de estas alumnas.

\section{Método}

Para dar respuesta a los objetivos establecimos un diseño de investigación de corte mixto, con técnicas cuantitativas y cualitativas, lo cual nos posibilitó generalizar resultados (cuantitativo) y profundizar en los datos contextualizando la información gracias a la cercanía con el entorno (cualitativo). Por lo tanto, la utilización del enfoque mixto favoreció la triangulación de los resultados para una mejor comprensión e interpretación del fenómeno estudiado.

\section{Muestra}

Los/as participantes del estudio fueron el profesorado y educadores/as sociales de cuatro centros de Sevilla, los cuales fueron elegidos por dos características comunes: 1) ubicación de la escuela en barrios de exclusión social, caracterizada por una población que tiene escasos recursos económicos, alta tasa de desempleo, escasa formación y cualificación; 2) alto porcentaje de alumnado gitano en la escuela. Los centros seleccionados fueron: CEIP Andalucía, CEIP Menendez Pidal, CEIP San José Obrero, CEIP María Zambrano. 
A continuación se presenta un perfil de la muestra del profesorado que participaron en el estudio (ver tabla 1):

Tabla 1

Perfil de la muestra del cuestionario. Profesorado.

\begin{tabular}{cccccc}
\hline \multicolumn{2}{c}{ Sexo } & \multicolumn{2}{c}{ Centro } & \multicolumn{2}{c}{ Titulación } \\
\hline \multirow{2}{*}{ Hombres } & $25,50 \%$ & CEIP Andalucía & $39.60 \%$ & Educ. Infantil & $8.00 \%$ \\
& & CEIP Menendez Pidal & $22.60 \%$ & Educ. Primaria & $48.00 \%$ \\
\hline \multirow{2}{*}{ Mujeres } & $74,50 \%$ & CEIP María Zambrano & $11.30 \%$ & Lengua Extranjera & $16.00 \%$ \\
\cline { 3 - 5 } & & CEIP San José Obrero & $26.40 \%$ & Educ. Física & $4.00 \%$ \\
& & & Educ. Especializada & $2400 \%$ \\
\hline
\end{tabular}

Fuente. Elaboración propia.

También participaron 6 educadores/as sociales, 2 Directores/as de los centros educativos, 3 jefas de estudio, 2 educadores/as de la Fundación del Secretariado Gitano (FSG) y 2 educadores/as de la Federación Andaluza de Mujeres Gitana (Fakali).

\section{Técnicas e Instrumentos}

Las técnicas de investigación empleadas fueron la encuesta y los grupos de discusión. Para la técnica de encuesta se utilizó un cuestionario formado por 80 ítems estructurado en las siguientes categorías: Identidad cultural y desventaja social; exclusión social / pobreza; vulnerabilidad; la situación de las mujeres gitanas en el contexto educativo (abandono escolar); formación docente; metodologías y estrategias de trabajo; recursos; y relación de la comunidad, familia, escuela. El proceso de validación del cuestionario se llevó a cabo a través de un formulario de revision cumplimentado por los/as educadores/as sociales de los centros educativos implicados, así como por profesionales de la FSG y de Fakali, los cuales modificaron algunas preguntas y añadieron algunas cuestiones que consideraron de intéres. Los resultados de los cuestionarios se analizaron con el paquete estadístico SPSS.

Por otro lado, se llevaron a cabo dos Grupos de Discusión en los que participaron profesores/as y educadores/as sociales de los centros implicados, así como profesionales de la FSG y de Fakali. Para el desarrollo de los grupos de discusión se utilizó un guión con preguntas centradas en: dificultades de las alumnas gitanas, participación de las familias en el centro, expectativas educativas de las familias, preparación y formación del profesorado, estrategias utilizadas por el centro. Las intervenciones fueron grabadas y transcritas, posteriormente se analizaron las transcripciones en función de las categorías del cuestionario. 


\section{Resultados}

\section{Perfil de las alumnas gitanas}

La mayoría del profesorado (98.10\%) nos indicó que las alumnas gitanas de sus centros educativos suelen proceder de niveles socioeconómicos bajos o muy bajos, esto se corresponde con el contexto en el que se sitúan los centros educativos analizados, algunos de los cuales se encuentran en zonas de alta vulnerabilidad social. Este contexto vulnerable también se aprecia en las respuestas del profesorado encuestado ya que el $42 \%$ del profesorado manifestó que sus alumnas suelen vivir en chabolas (vivienda de escasas proporciones y pobre construcción en zonas de exclusión social), el $42 \%$ indican que viven en pisos, y el resto (16\%) indican que viven itinerantes entre chabolas y pisos, sin una ubicación fija.

Los grupos de discusión confirman que el alumnado objeto del presente estudio proviene de zonas de marginación y aunque vivan en pisos se produce el denominado "chabolismo vertical". En palabras de una educadora social "es una situación de chabolismo vertical no reconocida, pero que es chabolista total, conviven con animales conviven con aguas fecales, en fin, una serie de historias que hay alli, en todas las plazoletas del cole" (A1). En la misma línea se manifiesta M1 "lo que tenemos es un alumnado que vienen también de los pisos que están hechos polvo, vamos a ver, chabolismo vertical". En la ciudad de Sevilla nos encontramos diversas situaciones en las que la vulnerabilidad y la exclusión social están relacionadas con las condiciones de las viviendas, son denominadas infraviviendas con problemas de salubridad o "chabolismo vertical", generando la segregación y la exclusión de las personas que viven en estas zonas.

Por todo ello, muchas de las dificultades que manifiestan el profesorado encuestado están más relacionadas con esta situación de exclusión que con la cultura gitana en sí. En esta misma línea los centros participantes en el estudio atienden a las necesidades del alumnado gitano no por la cuestión cultural, sino porque todos ellos provienen de contextos de exclusión y marginación social, de esta forma manifestaron los/as educadores/as sociales que la situación socioeconómica de las alumnas determina mucho más el proceso educativo que la cultura gitana. La mayoría de las veces el alumnado presenta conductas que están más relacionadas con la cultura de la marginación que con la cultura gitana: "Las adaptaciones que hacemos en el cole, no la hacemos porque el alumnado sea gitano, sino por un alumnado que vive en una situación de exclusión" (Grupo de Discusión, A1). En el mismo sentido nos indica M1 "nuestra población también es mayoritariamente gitana, pero pasa lo mismo, los payos que tenemos tienen la misma cultura, la cultura de la exclusión".

La misma educadora social nos indica que "nada de lo que hacemos en el centro lo hacemos porque el alumnado es gitano, que lo es, sino porque vienen de una situación de exclusión social", por lo tanto, podemos afirmar que la cultura gitana en sí no causa desventajas educativas, es la situación de exclusión social en la que se encuentran algunos grupos lo que incide de forma negativa en el proceso educativo del alumnado gitano, es decir, no es la cultura gitana sino la situación de exclusión y marginación social que se experimenta en el contexto estudiado. En este sentido coincidimos con Padilla, GonzálezMonteagudo y Soria-Vilchez (2017, p. 193) al considerar que es fundamental "separar cultura gitana a cultura de marginación". 
En esta misma línea, Gil (2017) establece la relación entre el nivel socioeconómico y los resultados educativos, e indica que los centros escolares pueden contribuir a compensar las desigualdades de partida del alumnado, poniendo en práctica medios y estrategias para paliar sus efectos.

\section{Expectativas Educativas y Proyección de Futuro de las alumnas gitanas}

Al preguntar al profesorado por las expectativas de las alumnas gitanas, nos indican que sus alumnas tienen como expectativa futura ayudar en casa y casarse. Paralelemante, el profesorado nos indica que las familias gitanas hace distinción entre los niños y las niñas, ya que quieren que sus hijos estudien hasta que trabajen y sus hijas hasta el matrimonio o hasta alcanzar los estudios obligatorios.

En relación a la actitud que presentan las familias gitanas sobre la educación de sus hijas, sólo el $31.4 \%$ del profesorado sostiene que para las familias es importante la educación de sus hijas, el resto sostiene que las familias no le dan importancia a la educación de sus hijas (ver tabla 2), ya que no les servirá para:

Tabla 2

Funcionalidad de la educación para las familias gitanas

\begin{tabular}{lc}
\hline $\begin{array}{l}\text { Las familias gitanas creen que la educación } \\
\text { no les sirve a sus hijas para... }\end{array}$ & $\begin{array}{c}\text { Porcentaje } \\
\text { del profesorado }\end{array}$ \\
\hline Alcanzar una titulación superior & $90.60 \%$ \\
Conseguir mejores trabajos & $75.50 \%$ \\
Alcanzar una titulación media & $79.20 \%$ \\
Adquirir más conocimientos & $66.00 \%$ \\
\hline
\end{tabular}

Fuente. Elaboración propia

Por otro lado, en los grupos de discusión se detacó la problemática existente con las niñas gitanas en el tránsito de secundaria a bachillerato, sobre todo a los 15 - 16 años de edad, momento en el que las niñas gitanas suelen abandonar sus estudios. Según los resultados de los grupos de discusión:

"Hemos conseguido retrasar la edad de abandono a los 16, el problema es que hay determinadas niñas que cuando llegan a los 16 hay familias que les impiden, que no les dejan terminar porque ya tienen edad de casarse, tiene edad ya de cuidar de la abuela o de la madre, también son familias que los padres han muerto o están en prisión. No hemos conseguido que continúen el bachillerato." (M1)

\section{Relación Familia - Centro Educativo}

Una de las cuestiones más importantes para el éxito de las alumnas gitanas en su proceso educativo es la relación de la familia con la escuela. Según el profesorado encuestado, las familias de las alumnas gitanas no suelen comunicar la falta de asis- 
tencia de sus hijas a clase, no suelen asistir a tutorías, casi nunca solicitan información sobre el proceso educativo de sus hijas, existe una baja participación de las familias en los órganos de gobierno, aunque indican que en general están satisfechas con el funcionamiento del centro y con el/la tutor/a de sus hijas (tabla 3).

Tabla 3

Relación de las familias con el centro educativo

\begin{tabular}{lcc}
\hline & NO & SI \\
\hline Las familias comunican las faltas de asistencia de sus hijas & $\mathbf{6 7 . 4 0 \%}$ & $32.60 \%$ \\
Las familias asisten a tutorías & $\mathbf{7 9 . 6 0 \%}$ & $\mathbf{2 0 . 4 0 \%}$ \\
Las familias dan importancia a la educación de sus hijas & $\mathbf{7 7 . 4 0 \%}$ & $\mathbf{2 2 . 5 0 \%}$ \\
Las familias solicitan información sobre el proceso educativo de sus hijas & $\mathbf{9 6 . 0 0 \%}$ & $4.10 \%$ \\
Las familias participan en los órganos de participación del centro educativo & $\mathbf{7 3 . 3 0 \%}$ & $\mathbf{2 6 . 7 0 \%}$ \\
Las familias están satisfechas con el funcionamiento del centro & $18.60 \%$ & $\mathbf{8 1 . 3 0 \%}$ \\
Las familias están satisfechas con el/la tutor/a de sus hijas & $\mathbf{1 7 . 1 0 \%}$ & $\mathbf{8 3 . 0 0 \%}$ \\
\hline
\end{tabular}

Fuente. Elaboración Propia

Sin embargo, a través de los grupos de discusión se indica que la participación de estas familias se realiza con estructuras diferentes a las tradicionales (tutoría), ya que se crean distintas estructuras de participación familiar con el objetivo de fomentar una participación más global.

"Tenemos una relación muy cercana y de confianza con nuestras familias. También es verdad que somos un centro de puertas abiertas, que nuestras familias entran desde por la mañana con los niños hasta las mismísimas clases. Se quedan un rato con las maestras, participan incluso en las Asambleas de clase, se quedan muchas de ellas voluntarias dentro del aula, a trabajar con los alumnos y con el profesor, entonces la verdad que nosotros sí que es una relación muy cercana y de confianza. Después tenemos creadas unas estructuras de participación y de organización dentro del centro, tenemos una Junta de familiares, tenemos talleres todos los días de la semana por la mañana, donde las familias que quieran pues acuden a realizar esos talleres." (A2)

De hecho, no existe una hora específica de atención a las familias, ni la formación hacia las familias se desarrollan fuera del horario lectivo del colegio, sino que se imparte en paralelo al horario de clase de sus hijos/as para facilitar la participación sobre todo de las madres:

"(...) hay un espacio en el cole, que es de adultas, que es para ellas, y que no van porque soy la madre de fulanito, yo voy porque soy una mujer. Entonces yo creo que esa es una clave muy importante. Creo que eso es importante, no solamente la relación con la familia como padre o madre de un alumno, sino pues una relación de participación de cómo entre todos mejoramos este cole." (A1) 
En la misma línea nos manifiestan educadores/as sociales de otro de los centros educativos, planteando que en estos centros se entiende la participación de forma muy diferente ya que no se utilizan las estructurales formales de espacio para tutorías, lo que no significa que no haya comunicación con las familias, sólo que se utilizan otras formas y estructuras.

"En el centro se ve como la gran asignatura pendiente, el tema de la participación de la familia. Realmente las tutorías las hacemos en las puertas a las nueve menos cuarto, de nueve menos cuarto a nueve y diez, y a la salida. Que es cuando se aprovecha, cuando hablan, cuando se dialoga, cuando le dices que tienes que venir a por las notas, cuando se da esa tutoría digamos no formal. Es otra forma de ver lo que es la participación de entender lo que es la participación. (...) Pero yo creo que sí hay participación y comunicación con los padres, pero no como entendemos que es en otros sitios, y la verdad que hay que buscar esas otras fórmulas." (M1).

En relación a la satisfacción de las familias, en los grupos de discusión nos indican que gran parte de la satisfacción de las familias con estos centros educativos se debe a la funcionalidad de los centros en sí. Son centros que han transcendido su función dentro de la educación formal y se han convertido en un recurso importante del barrio para las familias. Los centros educativos de contextos marginales se constituyen en un pilar básico para la detección de necesidades y para la gestión de los recursos en el barrio.

"Os habéis convertido en fuentes de recursos, los colegios ahora mismo, son el recurso principal, sobre todo en barrios excluidos, del que tiran sustituyendo a unos servicios sociales. Porque la gente viene más a vernos a nosotros que a unos Servicios Sociales, o en vez de ir al Centro de adultos (...) nos ven como mucho más cercanos" (A3).

"Te vienen para decirte oye búscame un curso, donde puedo ir apuntarme, donde tengo que ir para, donde me saco el bonobús, pídeme una cita para la ayuda, pídeme, habla con Servicios Sociales a ver si me atienden. Ósea que estamos haciendo ese tipo de mediación". (M1).

Los resultados obtenidos coinciden con los manifestados en otras investigaciones. Márquez y Padua (2016) resaltan que es fundamental que el profesorado se implique en el contexto y a su vez que la familia lo haga en el centro. Según ambas autoras "La construcción de un modelo de participación democrática junto con las familias y los estudiantes es el inicio de un modo de relación pedagógica orientado a las posibilidades y al cambio de expectativas de todas las partes" (2016, p. 99). En esta línea, Collet-Sabé, Besalú, Feu y Tort (2014, p. 24) proponen como primer eje de trabajo para el éxito escolar, especialmente en contextos más desfavorecidos, un modelo de relaciones en el que la familia participe activamente en la escuela. Como primer responsable para incentivar, motivar y activar la construcción de "un país-escuela inclusivo, democrático y para todos los niños y niñas y sus familias (ciudadanía)" se encuentra el o la docente.

Sobre la implicación de las familias de las niñas gitanas en los órganos de participación de la escuela, el 73,3\% del profesorado sostienen que la participación de las familias es muy baja. Para favorecer la participación de las familias gitanas en el centro educativo 
es necesario establecer otros mecanismos de participación que transciendan los cauces formales, crear estrategias de participación cercanas a su cultura, flexible y que permita ser escuchados. En ese sentido nos plantea un educador social en los grupos de discusión:

"Creo que es básico también darles voz a esas personas de esa otra cultura, y darle voz es que puedan participar en el centro, que puedan proponer actividades en el centro y que de alguna manera consigamos que el centro lo sientan como suyo, con la libertad de proponer. (...), hacerla visible en los centros, hacerles participe en los centros, buscando los espacios, buscando las estructuras y dedicándole el tiempo." (A1).

\section{Rendimiento académico y necesidades educativas de las alumnas gitanas}

En cuanto a la prioridad que las alumnas gitanas tienen hacia algunas cuestiones educativas podemos ver que el $47 \%$ del profesorado indican que para ellas no es prioritario su rendimiento académico, que no se esfuerzan ni tienen dedicación (52\%), y que prestan poca atención en clase $(38.5 \%)$.

Sobre el rendimiento académico, el $48.9 \%$ del profesorado manifiestan que las alumnas gitanas no aprueban todas las asignaturas, y suelen suspender las asignaturas obligatorias. A su vez, el 68.7\% manifiestan que las alumnas gitanas no realizan las tareas y deberes en casa porque tienen falta de apoyo familiar (37.5\%) o por otras razones familiares (55\%). A su vez, el 69.8\% del profesorado manifiesta que a las alumnas gitanas no le gusta estudiar y el 66\% indican que asisten desmotivadas a la escuela. Es importante tener en cuenta las creencias del profesorado ya que puede influir en el rendimiento académico de su alumnado de acuerdo con la teoría del efecto Pigmalión o profecía autocumplida.

Sobre las necesidades educativas de las alumnas gitanas el profesorado indica que las alumnas gitanas presentan déficit educativo principalmente en las áreas de lenguaje, comprensión lectora, matemáticas y conocimiento del medio (tabla 4):

Tabla 4

Necesidades educativas de las alumnas gitanas

\begin{tabular}{l|c}
\hline & Porcentaje de profesorado \\
\hline Lenguaje & $75.50 \%$ \\
Comprensión Lectora & $75.00 \%$ \\
Razonamiento Matemático & $61.50 \%$ \\
Comprensión del Medio & $66.70 \%$ \\
Educación Física & $25.50 \%$ \\
\hline
\end{tabular}

Fuente. Elaboración Propia

Según Cordero, Pedraja y Simancas (2015) existen numerosos estudios donde se comprueban la relevancia del nivel socioeconómico de las familias en los resultados académicos del alumnado. Estos autores presentan una serie de datos que determinan la importancia del contexto socioeconómico en los resultados académicos del alumnado, y establecen una serie de factores para mejorar el progreso del alumnado proveniente 
de contextos de exclusión social entre los que destacamos la calidad docente para fomentar la motivación del alumnado, el cual debe superar las adversidades de un entorno socieconómico desfavorable.

A través de los grupos de discusión hemos podido comprobar la importancia de educar en la emoción como metodología docente, sobre todo en niños y niñas provenientes de contextos desfavorecidos y de zonas de alta vulnerabilidad social. Para ello, no basta con medir el rendimiento académico de los/as estudiantes, es importante educar en la resiliencia y la afectividad, sobre todo en las alumnas gitanas como base para su empoderamiento. Por ello los/as educadores/as sociales de los centros educativos estudiados utilizan la emoción y la efectividad como herramienta didáctica para promover la cohesión social y el sentimiento de pertenencia al centro educativo.

"La palabra resiliencia que es la palabra donde realmente, resiliencia y emotividad. Resiliencia en cuanto al profesorado, que es capaz de abrazar al alumno que tiene problemas, y que lo asume como propio. Y que se identifica. Se identifica con que ese alumnado es parte del cole (...) Esa identificación, ese trabajo que el profesor hace día a día con el alumnado, porque no son alumnos con nombres y apellidos y ya está, son niños con corazón y con sentimientos, eso es fundamental. Es decir, la conciencia de grupo. Nuestros niños son niños muy necesitados de afectividad. Por eso la emotividad es fundamental. Y ese es el engranaje que hace que el colegio vaya funcionando." (J1).

Cuando hemos preguntado al profesorado sobre qué elementos influyen en el proceso educativo de las alumnas gitanas, la mayoría sostienen que influye la participación de las familias en la escuela (72.6\%), el nivel educativo de las familias (62\%), la formación del profesorado (60\%), y el nivel económico de las familias (56.9\%). Aunque en menor porcentaje, existe un $41.1 \%$ del profesorado que indican que el pertenecer a la cultura gitana, sobre todo siendo mujer, influye de forma negativa en el proceso educativo de estas niñas.

Según el profesorado, para mejorar el proceso educativo de las alumnas gitanas es fundamentar-mejorar los procesos de orientación educativa y el diseño de un plan de acción tutorial individualizado que contemple (tabla 5):

Tabla 5

Cuestiones importantes para la mejora del proceso educativo

\begin{tabular}{lc}
\hline & Porcentaje de profesorado \\
\hline Coordinación con otros agentes y servicios & $90.30 \%$ \\
Aulas de Apoyo & $83.80 \%$ \\
Diagnóstico individualizado & $81.80 \%$ \\
Plan curricular individualizado & $77.50 \%$ \\
Orientación individualizada & $73.20 \%$ \\
Orientación familiar & $70.60 \%$ \\
Cultura gitana en el centro y en el curriculum & $53.30 \%$ \\
\hline
\end{tabular}

Fuente. Elaboración propia 
Según la información de los grupos de discusión, además de las cuestiones indicadas en la tabla 5, es fundamental contar con referentes positivos de mujeres gitanas que actúen como modelo dentro de su comunidad, es decir, mujeres gitanas que hayan terminado su formación y estén insertas en el mundo laboral. Sin embargo, a día de hoy en estos contextos de marginación y alta vulnerabilidad social, existen pocos referentes que sirvan de guía a las generaciones más jóvenes. En este sentido, nos comentan educadores/as de los centros educativos participantes:

"Nos falta sobre todo tener referentes, sobre todo referentes positivos en esas familias (...) y en el barrio, mira la amiga de tu hermana, ella sí tiene estudios. Y eso es lo que nos falta." (M1)

"Nosotros tenemos una antigua alumna del cole estudiando el último curso de grado de magisterio, y ya está contratada por nosotros para actividades extraescolares, y la ven como entra una niña gitana para ayudar en las actividades extraescolares, y se quedan diciendo si ésta es mi vecina. Y ese tipo de ejemplos sí les llega, entonces hasta que no vean esos ejemplos, hasta que nosotros mismos no sepamos trasmitirles esos ejemplos no, porque hay muchas barreras, embarazos, género, institutos, profesorado, no sé cuánto, demasiadas." (A3)

\section{Centros Educativos y Formación Docente}

Al preguntar si la cultura gitana está presente en el currículum del centro educativo, el $66 \%$ del profesorado sostiene que sí, aunque el $13.7 \%$ indican que no está presente pero que sería necesario. En ese sentido, tres de los centros que han participado en esta investigación indican que ellos tienen los elementos de la cultura gitana integrados en su centro, tanto en el Plan de Centro, como en el currículum y en el Plan de Convivencia.

"...la cultura gitana está presente en el centro y es visible en el centro, no es anecdótica. Nosotros tenemos muchos temas alrededor de lo que es la cultura gitana, desde las normas que están redactadas por escrito, las redactó el alumnado junto con las familias y el profesorado, y una norma escrita en la norma de convivencia es: se respetan los muertos de toda la comunidad, porque el respeto de los muertos es básico e importante para la población que tenemos. Entonces, el incluir esa cultura, en nuestro caso gitana, es básico (...) porque cuando llegan al centro lo gitano está presente, y está presente no solamente simbólicamente porque hay carteles, porque esté la bandera, porque esté himno, porque hacemos el día del gitano andaluz, el día del pueblo gitano mundial. (...) Y de alguna manera eso se tiene que ver reflejado en el currículo que hacen los alumnos dentro del aula." (A1).

En relación a las medidas que se realizan en los centros educativos, indicamos aquellas que han obtenido mayores porcentajes, las cuales coinciden con las medidas que consideran necesarias para el buen proceso educativo de las alumnas gitanas:

- Coordinación con otros agentes y servicios: 97.7\%.

- Diagnóstico individual de necesidades: 95.5\%. 
- Orientación educativa a las alumnas gitanas que lo necesiten: $95.3 \%$.

- Plan individualizado: $92.9 \%$.

- Orientación educativa a las familias de las alumnas gitanas que lo necesiten: $90 \%$.

- Aulas de Apoyo: 56.5\%.

Como dato a destacar debemos señalar que el 64.2\% del profesorado encuestado han manifestado no haber recibido ningún tipo de formación sobre la cultura gitana, lo que consideramos un porcentaje elevado, el resto ha recibido algo de formación en cursos de formación permanente o en cursos propios del centro educativo. Sin embargo, en los grupos de discusión se manifestó la importancia que tiene para el profesorado de estos centros la formación recibida sobre la cultura gitana, para comprender y entender mejor a la comunidad educativa y al contexto donde se inscribe el centro educativo.

"Hemos tenido unas jornadas con la asociación (...), y hemos estado todo el profesorado en esas jornadas hablando precisamente del alumnado gitano en la escuela. $Y$ nos resultó muy interesante, creo que es una buena idea el introducir este tipo de formación docente" (J3).

A su vez, el 83\% del profesorado sostiene que no ha recibido formación específica sobre Educación Intercultural, y la poca formación que han recibido ha sido por parte de la Universidad o en cursos de formación del profesorado. El 81.4\% del profesorado manifiesta que debe seguir formándose en Educación Intercultural ya que tiene carencias formativas para trabajar con este colectivo.

\section{Discusión y Conclusiones}

En síntesis, podemos resaltar la importancia de tomar como referente a la mujer gitana ya que, tal y como nos indica la Fundación para el Secretariado Gitano (2000), ella es el eje de organización familiar y de responsabilidad en las alternativas educativas de sus miembros. Si bien es importante trabajar con toda la familia, la mujer es la que más participa en el contexto educativo, la que suele acudir a la escuela y la que se responsabiliza de los hábitos y rutinas escolares.

A su vez, desde la escuela se debe tener presente las etapas evolutivas de las niñas gitanas, ya que empiezan a asumir a edad más temprana roles de la etapa adulta, y esta cuestión debe ser tenida en cuenta por la escuela para favorecer la permanencia de la alumna gitana en el sistema educativo, sobre todo en Educación Secundaria, siendo ésta una de las obligaciones principales del centro, pues desde una perspectiva oficial, el colegio debe favorecer la adaptación cultural de sus estudiantes (Jiménez, Fardella, \& Muñoz, 2017). Podemos afirmar que se ha conseguido erradicar el absentismo en la Educación Primaria, sin embargo, en las etapas posteriores son las que las alumnas gitanas suelen presentar mayor abandono escolar, sobre todo a los 15-16 años de edad, algunas incluso no terminan la etapa escolar obligatoria (hasta los 16 años) ya que las familias consideran que es la edad en la que tienen que casarse o ocuparse de la familia y de la casa. Por lo tanto, el abandono escolar de las alumnas gitanas en secundaria y en bachillerato es una de las cuestiones que se debe abordar. 
En cuanto al rendimiento de las alumnas gitanas y a su progreso en la escuela, podemos establecer que suelen tener dificultades en las materias básicas como lenguaje, comprensión lectora, matemáticas y conocimiento del medio; suelen suspender estas asignaturas y no suelen hacer las tareas. Por ello es fundamental establecer un plan de acción tutorial individualizado y familiar que realice un diagnóstico de las necesidades que presenta cada alumna para poder intervenir con ella y con su familia, reforzar las aulas de apoyo y establecer una coordinación con otras entidades y servicios del contexto. En definitiva, trabajar por una escuela basada en el modelo de comunidades de aprendizaje en el que participan todos los agentes de la Comunidad Educativa: Profesorado, Alumnado, Familias, Entidades Locales.

Otra de las cuestiones que destacamos es que las familias gitanas tienen pocas expectativas relacionadas con la educación de sus hijas entendiendo que la educación no les sirve para mucho ya que su proyección de futuro es cuidar de la familia o casarse cuando cumplan 16 años, por lo que esperan que estudien hasta terminar la educación secundaria obligatoria produciéndose un abandono de los estudios en esa etapa. Por ello es importante insistir en la necesidad de reforzar los procesos de orientación, incluida la orientación familiar para favorecer que las alumnas gitanas continúen sus estudios y las apoye en su proceso educativo.

Un elemento que consideramos importante para la permanencia de las alumnas gitanas en el sistema educativo es incorporar al Plan de Centro y al Currículum aspectos y elementos cercanos a su vida y a sus expectativas, trabajar para que lo aprendido en la escuela lo consideren útil para su desarrollo vital. Según el profesorado entrevistado, la mayoría de las familias consideran que sus hijas deben aprender las tareas de la casa y deben estudiar hasta el matrimonio, por lo que no ven utilidad en el sistema educativo, los resultados nos indican que no creen que la educación les posibilite a sus hijas una mejor vida ya que su futuro será casarse y no trabajar. Es necesario motivar a las familias para que vean la escuela útil para el desarrollo de sus hijas, y esto puede realizarse introduciendo en el currículum cuestiones cercanas a su vida y referentes cercanos.

Ya existen experiencias en algunos de los centros educativos que han participado en la investigación de la incorporación de referentes femeninos gitanos en la escuela, es decir, mujeres gitanas que han estudiado y se desarrollan profesionalmente dentro del ámbito educativo, sirviendo de apoyo en la educación de las niñas gitanas y un modelo a seguir. Es importante seguir contando con estos referentes femeninos para poder visibilizar la funcionalidad de la educación como motor de cambio, pudiendo provocar cambios en las expectativas familiares de las niñas gitanas. Tal y como nos indican en la investigación "es importante cuando te ven las niñas o sus madres y dicen "mira mi profe es gitana, o es mi vecina, yo quiero ser como ella»" (educadora social gitana participante en el grupo de discusión).

Los centros educativos analizados establecen estrategias de comunicación con las familias diferentes a las convencionales. Por ello, a pesar de que los resultados del cuestionario indiquen que las familias no asisten a tutorías esto no quiere decir que no exista comunicación, ya que se dan procesos de tutorización adaptados a los horarios y a procesos de las familias que conforman la comunidad educativa. En 
nuestro estudio hemos puesto de manifiesto que en estos centros se establece un modelo de participación democrática donde el eje es el centro educativo, favoreciendo la participación de las familias en la escuela y en su comunidad.

En la misma línea, afirmamos que los centros educativos que se ubican en zonas de alta vulnerabilidad social son un recurso imprescindibles para la comunidad y para las familias, ya que su función transciende a la función escolar y se convierten en centros de atención directa a familias con necesidades socioeconómicas. Las familias suelen acudir a estos centros educativos para resolver problemas y situaciones no escolares, esto es debido a las fuertes estructuras de comunicación que se crean y a la confianza que depositan en el profesorado y en los agentes educativos de las escuelas.

Por último, queremos señalar que el alumnado gitano con dificultades educativas suele presentar desventajas socioeconómicas. No podemos confundir las cuestiones culturales con la marginación socioeconómica que suelen encontrarse gran parte de las personas pertenecientes a minorías culturales, ni confundir los valores culturales con los que normalmente se identifican estas minorías con las consecuencias producidas por la marginación, la pobreza y la exclusión social. Y muchos de los problemas educativos del alumnado gitano están más relacionados con la pobreza y la exclusión social que con la cultura, siendo esta una de las cuestiones que más han salido a lo largo del proceso de investigación.

Desde nuestro planteamiento la escuela responde a los valores y a las necesidades de una cultura mayoritaria, y tiende a homogeneizar impartiendo la docencia en una sola lengua, con los mismos objetivos establecidos, con una misma metodología, una misma visión del mundo y de cómo entenderlo, etc. Aún a día de hoy donde la diversidad cultural es un hecho y reconocida en el ámbito educativo, la atención a la diversidad cultural del alumnado gitano sigue siendo una asignatura pendiente, aunque se están desarrollando experiencias educativas que a lo largo del tiempo van demostrando su efectividad.

\section{Referencias}

Asensio, A. (2016). Educación y población gitana. Avances en Supervisión Educativa, 25, 1-34. Recuperado de https://avances.adide.org/index.php/ase/article/view/547/390

Ayala Rubio, A. (2014). La resocialización de la mujer gitana en los cursos de alfabetización de la Renta Mínima de Inserción. Política y Sociedad, 51(3), 789-816. Recuperado de http://revistas.ucm.es/index.php/POSO/article/view/43725/44512

Collet-Sabé, J., Besalú; X., Feu, J., \& Tort, A. (2014). Escuelas, familias y resultados académicos. Un nuevo modelo de análisis de las relaciones entre docentes y progenitores para el éxito de todo alumnado, Profesorado. Revista de currículum y formación del profesorado, 18(2), 8-33. Recuperado de http://www.ugr.es/local/recfpro/rev182ART1.pdf

Cordero Ferrera, J.M., Pedraja Chaparro, F., \& Simancas Rodríguez, R. (2015). Factores del éxito escolar en condiciones socioeconómicas desfavorables. Revista de Educación, 370, 172-198. doi: 10.4438/1988-592X-RE-2015-370-302

El-Habib Draoui, B., Jiménez-Delgado, M., Ruiz-Callado, R., \& Jareño-Ruiz, D. (2016). Composición escolar y expectativas del alumnado por origen y etnia: una aproxima- 
ción a la segregación escolar extraoficial. RASE: Revista de la Asociación de Sociología de la Educación, 9(1), 59-77. Recuperado de http://rua.ua.es/dspace/handle/10045/52788

Fundación para el Secretariado Gitano (2000). Mujeres Gitanas. FSG, (1). Recuperado de https://www.gitanos.org/upload/09/93/Rev_1_._05_-_Dossier.pdf

Fundación para el Secretariado Gitano (2010). Evaluación de la normalización educativa de las alumnas y los alumnos gitanos en Educación Primaria. Madrid, IFIIE/ Instituto de la Mujer.

Fundación para el Secretariado Gitano (2013). Resumen ejecutivo. El alumnado gitano en Secundaria. Un estudio comparado. Ministerio de Educación, Cultura y Deporte. Recuperado de https://www.gitanos.org/upload/92/20/EstudioSecundaria.pdf

Gil Flores, J. (2017). Características del profesorado y desempeño docente en aulas con alumnado de bajo nivel socioeconómico. Revista de Investigación Educativa, 35(1), 133-150. doi: http://dx.doi.org/10.6018/rie.35.1.246381

Jiménez Vargas, F., Fardella Cisternas, C., \& Muñoz Proto, C. (2017). Una aproximación microetnográfica de prácticas pedagógicas en escuelas multiculturales. Tensiones y desafíos en torno a la escolarización de inmigrantes y grupos minoritarios. Perfiles educativos, 39(156), 72-88. Recuperado de http://perfileseducativos.unam.mx/iisue_pe/ index.php/perfiles/article/view/58284

Llevot, N., \& Bernad, O. (2016). La mediación gitana: herramienta performativa de las relaciones entre escuela y familia. Revista electrónica interuniversitaria de formación del profesorado, 19(1), 99-110. doi: http://dx.doi.org/10.6018/reifop.19.1.245581

López Rodriguez, M. E., \& Sanz Hernández, A. (2017). Reflexión, acción, decisión: trayectorias en la construcción de la identidad de género en el patriarcado gitano. EMPIRIA. Revista de Metodología de las Ciencias Sociales, 38, 41-62. doi: https:// doi.org/10.5944/empiria.38.2018.19705

Macías, F., \& Redondo, G. (2012). Pueblo gitano, Género y Educación: investigar para excluir o investigar para transformer. International Journal of Sociology of Education, 1(1), 71-92. doi: http://dx.doi.org/10.4471/rise.2012.04

Márquez, M J., \& Padua, D. (2016). Comunidad Gitana y Educación Pública. La necesidad de construir un proyecto social y educativo compartido. Revista Interuniversitaria de Formación del Profesorado, 30(1). Recuperado de http://www.redalyc.org/articulo. oa?id=27446519009

Márquez, M.J, Prados, M.E, \& Padua, D. (2017). La voz de Tsura. Un relato biográficonarrativo sobre mediación intercultural y su sentido resiliente en el ámbito educativo. Educar, 54(1), 49-66. Recuperado de https://ddd.uab.cat/record/184668

Ministerio de Sanidad, Seguridad Social e Igualdad [MSSSI]. (2012). Estrategia Nacional para la Inclusión del Pueblo Gitano en España (2012-2020). Recuperado de www.msssi.gob.es

Padilla Carmona, T., González-Monteagudo, J., \& Soria-Vílchez, A. (2017). Gitanos en la Universidad: Un estudio de caso de trayectorias de éxito en la Universidad de Sevilla. Revista de Educación, 377, 187-211. doi: https://doi.org/10.4438/1988592X-RE-2017-377-358

Parra Toro, I., Alvarez-Roldan, A., \& Gamella, J. F. (2017). Un conflicto silenciado: Procesos de segregación, retraso curricular y abandono escolar de los adolescentes gitanos. Revista de Paz y Conflictos, 10(1), 35-60. Recuperado de https://dialnet.unirioja.es/servlet/articulo?codigo $=6103275$ 
Rodríguez, A., \& Paterna, C. (2016). Programa de intervención psicosocial y educativa en habilidades sociales y género con adolescentes de etnia gitana. En Castejón Costa (coord.). Psicología y Educación: presente y future (2636-2644). ACIPE: Asociación Científica de Psicología y Educación. Recuperado de https://rua.ua.es/dspace/ bitstream/10045/64440/1/Psicologia-y-educacion_317.pdf

Sánchez-Muros, P.S. (2015). Minoría gitana y prejuicio étnico en la preadolescencia. Procesos de exclusión e inclusión en el ámbito escolar. RASE Revista de Sociología de Educación, 8(3), 396-408. Recuperado de https://ojs.uv.es/index.php/RASE/article/ view/8392/7985

Zugaza, U. (2017). Claves hacia el empoderamiento de las mujeres gitanas: un análisis desde el punto de vista de la interseccionalidad. Investigaciones feministas: papeles de estudios de mujeres, feministas y de género, 8(1), 203-222. doi: http://doi.org/10.5209/ INFE.54459

Fecha de recepción: 31 de marzo de 2018.

Fecha de revisión: 19 de abril de 2018.

Fecha de aceptación: 22 de junio de 2018. 
